OPEN ACCESS

Edited by:

Xihui Shen,

Northwest A\&F University, China

Reviewed by:

Zongmin Du,

Beijing Institute of Microbiology and Epidemiology, China

Bo Pang,

National Institute for Communicable

Disease Control and Prevention (China $C D C$ ), China

*Correspondence: Yi-Cheng Sun

sunyc@ipbcams.ac.cn

Received: 11 June 2017

Accepted: 25 July 2017

Published: 08 August 2017

Citation:

Ren G-X, Guo X-P and Sun Y-C (2017) HmsC Controls Yersinia pestis Biofilm Formation in Response to

Redox Environment.

Front. Cell. Infect. Microbiol. 7:355.

doi: 10.3389/fcimb.2017.00355

\section{HmsC Controls Yersinia pestis Biofilm Formation in Response to Redox Environment}

\author{
Gai-Xian Ren, Xiao-Peng Guo and Yi-Cheng Sun*
}

MOH Key Laboratory of Systems Biology of Pathogens, Institute of Pathogen Biology, Chinese Academy of Medical

Sciences and Peking Union Medical College, Beijing, China

Yersinia pestis biofilm formation, controlled by intracellular levels of the second messenger molecule cyclic diguanylate (c-di-GMP), is important for blockage-dependent plague transmission from fleas to mammals. HmsCDE is a tripartite signaling system that modulates intracellular c-di-GMP levels to regulate biofilm formation in $Y$. pestis. Previously, we found that $Y$. pestis biofilm formation is stimulated in reducing environments in an hmsCDE-dependent manner. However, the mechanism by which $H m s C D E$ senses the redox state remains elusive. Using a dsbA mutant and the addition of $\mathrm{Cu}^{2+}$ to simulate reducing and oxidizing periplasmic environments, we found that $\mathrm{HmsC}$ protein levels are decreased and the HmsC-HmsD protein-protein interaction is weakened in a reducing environment. In addition, we revealed that intraprotein disulphide bonds are critical for HmsC since breakage lowers protein stability and diminishes the interaction with $\mathrm{HmsD}$. Our results suggest that $\mathrm{HmsC}$ might play a major role in sensing the environmental changes.

Keywords: HmsC, biofilm formation, Yersinia pestis, HmsD, c-di-GMP

\section{INTRODUCTION}

Yersinia pestis, the causative agent of plague, is a Gram-negative bacterium that is transmitted to mammals via bites from infected fleas. Transmission of $Y$. pestis is greatly enhanced following formation of a bacterial biofilm in the proventriculus of the flea (Eisen et al., 2006, 2007; Eisen and Gage, 2009). Y. pestis biofilms are positively regulated by cyclic-di-GMP (c-di-GMP), a second messenger present in numerous Gram-negative bacteria. C-di-GMP is synthesized by diguanylate cyclase (DGC) enzymes and degraded by phosphodiesterase (PDE) enzymes (Ryjenkov et al., 2005; Schmidt et al., 2005; Ryan et al., 2006). The Y. pestis genome encodes two DGCs, HmsT and HmsD. HmsT plays a major role in biofilm formation in vitro, while HmsD plays a more prominent role in blocking proventricular biofilm formation in the flea (Jones et al., 1999; Kirillina et al., 2004; Bobrov et al., 2011; Sun et al., 2011).

HmsC, a periplasmic protein, represses HmsD via direct interaction (Ren et al., 2014; Bobrov et al., 2015), while HmsE, an outer membrane protein, counteracts with $\mathrm{HmsC}$ and activates $\mathrm{HmsD}$ (Bobrov et al., 2015). The hmsCDE operon is homologous to the $y f i B N R$ operon in Pseudomonas aeruginosa, which has been shown to regulate biofilm-dependent phenotypes (Malone et al., 2010, 2012). A reducing environment stimulates biofilm formation in an $y$ fiBRNR ( $h m s C D E$ )-dependent manner (Ren et al., 2016) Consistently, mutation of $d s b A$, encoding a periplasmic protein involved in creating an oxidizing environment by catalyzing disulfide bond formation, leads to increased biofilm formation in E. coli and Salmonella enteric (Anwar et al., 2014; Hufnagel et al., 2014). 
However, the mechanism by which the HmsCDE (YfiBRR) system senses the redox state and regulates biofilm formation is not clear.

DsbA and DsbB coordinate to generate an oxidizing environment in the periplasm (Bardwell et al., 1993). DsbB, an inner membrane protein, oxidizes DsbA, which in turn oxidizes periplasmic proteins by catalyzing disulphide bond formation (Bardwell, 1994; Nakamoto and Bardwell, 2004). The oxidant $\mathrm{Cu}^{2+}$ catalyzes periplasmic disulphide bond formation and rescues defects in periplasmic disulphide bond formation in $d s b A$ null strains (Battistoni et al., 1999). In the present study, we used a $d s b A$ mutant and the addition of $\mathrm{Cu}^{2+}$ to simulate reducing and oxidizing periplasmic environments, and analyzed the role of $\mathrm{HmsCDE}$. We found that in a reducing environment $\mathrm{HmsC}$ protein levels were decreased, and the interaction between $\mathrm{HmsC}$ and $\mathrm{HmsD}$ was weakened. Further analysis showed that $\mathrm{HmsC}$ contains four conserved cysteines that form two pairs of disulphide bonds that are important for protein stability and interaction with HmsD. Thus, we propose that HmsC senses the redox state in the periplasm, regulates $\mathrm{HmsD}$ in response and thereby controls biofilm formation in $Y$. pestis.

\section{MATERIALS AND METHODS}

\section{Experimental Procedures}

\section{Bacterial Strains and Plasmids}

The strains and plasmids used are listed in Table S1. The Y. pestis KIM6+ strain cured plasmid pCD1 was used as the wildtype strain. KIM6+ mutants were generated by inserting PCR products into the chromosome using the Red recombination system (Datsenko and Wanner, 2000; Sun et al., 2008). All strains and plasmids were verified by PCR, DNA sequencing and plasmid complementation, as appropriate.

\section{In vitro Biofilm Assays}

Microtiter plate biofilm assays were carried out as previously described with minor modifications (Sun et al., 2011). Briefly, cells were grown in LB broth supplemented with $4 \mathrm{mM}$ $\mathrm{MgCl}_{2}$ and $4 \mathrm{mM} \mathrm{CaCl}_{2}$ overnight at $26^{\circ} \mathrm{C}$, diluted to an $\mathrm{OD}_{600}$ of 0.05 using the same medium or the same medium supplemented with $0.8 \mathrm{mM} \mathrm{CuSO}$, aliquoted into 96-well polystyrene plates and incubated with shaking at $200 \mathrm{rpm}$ for $24 \mathrm{~h}$ at $26^{\circ} \mathrm{C}$. Plates were then washed three times with distilled water and adhered biofilms were stained with $0.01 \%$ crystal violet for $15 \mathrm{~min}$. The dye was later re-dissolved in $80 \%$ ethanol and $20 \%$ acetone, and the absorbance at 600 $\mathrm{nm}$ was measured. Results from four independent experiments with at least three replicates per experiment were analyzed by one-way analysis of variance (ANOVA) with Dunnett's post-test.

\section{Western Blotting}

Overnight cultures of $Y$. pestis were diluted 1,000-fold in 50 $\mathrm{ml} \mathrm{LB}$ broth at room temperature for $16 \mathrm{~h}$. Samples were extracted from the same amount of stationary phase cells cultured at $26^{\circ} \mathrm{C}(100 \mathrm{ng}$ total protein for detection of $\mathrm{HmsD}$ and
HmsE, $20 \mathrm{ng}$ total protein for detection of $\mathrm{HmsC}$ ), separated by 10 or $15 \%$ SDS-PAGE, transferred to a PVDF membrane, and analyzed by immunoblotting with antibodies to the HA tag (Sigma), the Flag tag (Invitrogen) or Myc (Invitrogen). Immobilon Western HRP Substrate (Millipore) was used for detection. Results were quantitated by densitometry using $\mathrm{NIH}$ Image J.

\section{Measurement of c-di-GMP Levels}

Intracellular c-di-GMP levels in $Y$. pestis were measured as previously described with minor modifications (Bobrov et al., 2011; Bellows et al., 2012; Ren et al., 2014). Overnight cultures of $Y$. pestis strains were diluted to an $\mathrm{OD}_{600}$ of $\sim 0.1$ and grown in $40 \mathrm{ml} \mathrm{LB}$ at $26^{\circ} \mathrm{C}$ to an $\mathrm{OD}_{600}$ of $\sim 0.8$. Cell pellets were collected and resuspended in $50 \mu \mathrm{l}$ extraction buffer $(40 \%$ methanol and $40 \%$ acetonitrile in $0.1 \mathrm{M}$ formic acid) per $48 \mathrm{mg}$ wet cell weight. Slurries were incubated for $30 \mathrm{~min}$ at $-20^{\circ} \mathrm{C}$ and insoluble material was removed by centrifugation at $4^{\circ} \mathrm{C}$. Supernatants were neutralized by the addition of $4 \mu \mathrm{l} 15 \%$ $\mathrm{NH}_{4} \mathrm{HCO}_{3}$ per $100 \mu \mathrm{l}$ sample and $10 \mu \mathrm{l}$ was analyzed using liquid chromatography tandem mass spectrometry. Synthetic c-di-GMP was used as a standard. Samples extracted from the $h m s T-h m s D$ double mutant were used as a negative control. Results from three independent experiments were analyzed using one-way ANOVA with Dunnett's post-test.

\section{In vivo Co-purification of $\mathrm{HmsC}$ and MBP-HmsD ${ }^{\text {N9-155 }}$}

Co-purification experiments were carried out as previously described (Ren et al., 2014). Briefly, the Y. pestis hmsCDE mutant expressing $\mathrm{P}-\mathrm{MBP}-\mathrm{HmsD}^{\mathrm{N} 49-155}$ with $\mathrm{HmsC}$ or $\mathrm{HmsC} \mathrm{C}^{\mathrm{C} 1 \mathrm{AC} 2 \mathrm{AC} 3 \mathrm{AC} 4 \mathrm{~A}}$ was inoculated into $200 \mathrm{ml}$ aliquots of LB medium supplemented with kanamycin $(30 \mu \mathrm{g} / \mathrm{ml})$ and ampicillin $(100 \mu \mathrm{g} / \mathrm{ml})$. Cells were grown to an $\mathrm{OD}_{600}$ of 0.6 and induced by addition of arabinose to a final concentration of $0.02 \%$. After induction for $12 \mathrm{~h}$ at $22^{\circ} \mathrm{C}$, cells were harvested by centrifugation, resuspended in $25 \mathrm{ml}$ PBS supplemented with protease inhibitor cocktail and lysed by sonication. After centrifugation at $10,000 \mathrm{~g}$ for $30 \mathrm{~min}$ at $4^{\circ} \mathrm{C}$, supernatants were purified by immobilized metal affinity chromatography using Ni-NTA resin. Purified proteins were collected and tested by western blotting.

\section{RESULTS}

\section{The Periplasmic Redox Environment Regulates $Y$. pestis Biofilm Formation in an hmsC-Dependent Manner}

Previously, we found that a reducing environment stimulates biofilm formation in an HmsCDE-dependent manner (Ren et al., 2016). To explore the mechanism by which HmsCDE senses the redox state and regulates biofilm formation, we first constructed a $d s b A$ null mutant to simulate a reducing environment. Biofilm formation was increased in the $d s b A$ mutant strain in an $\mathrm{HmsD}$-dependent manner (Figure 1A). In addition, biofilm formation also increased in the $d s b B$ mutant 
(Figure S1). Next, we added the oxidant $\mathrm{CuSO}_{4}$ to simulate an oxidizing environment. Addition of $\mathrm{CuSO}_{4}$ significantly repressed biofilm formation in the $d s b A$ mutant but not in the wild-type strain (Figure 1A). Finally, we found that addition of the reducing agent DTT was unable to increase $Y$. pestis biofilm formation when $d s b A$ was overexpressed (Figure S2), suggesting that a reducing environment could be overcome by the periplasmic oxidizing environment. Taken together, these results suggest that a reducing periplasmic environment caused by $d s b A$ mutation stimulates biofilm formation in $Y$. pestis and addition of the oxidant $\mathrm{CuSO}_{4}$ generates an oxidizing periplasmic environment that represses biofilm formation.

To further investigate the role of $h m s C D E$ in response to the redox environment, we constructed a series of $d s b A$ mutants and analyzed their biofilm formation. Compared with the super biofilm formation phenotype of the $d s b A$ mutant, biofilm formation in the $d s b A-h m s D$ double mutant was markedly decreased. However, in the $d s b A-h m s T$ and $d s b A-h m s E$ double mutant strains, biofilm formation was relatively unchanged compared with that in the $d s b A$ single mutant (Figure 1A), suggesting that $h m s T$ and $h m s E$ are not involved in sensing the redox environment. The super biofilm formation phenotype of the $d s b A$ mutant could be recovered by complementation with either $d s b A$ or $h m s C$, or addition of $\mathrm{CuSO}_{4}$ in Figures $\mathbf{1 A}, \mathbf{B}$. However, the super biofilm formation phenotype caused by $h m s C$ mutation could not be complemented by overexpression of $d s b A$ or addition of $\mathrm{CuSO}_{4}$ (Figure 1A, Figure S3), suggesting that $\mathrm{HmsC}$ might act as a sensor of the redox environment. Taken together, these results suggest that the redox periplasmic environment regulates $Y$. pestis biofilm formation in an $h m s C D E$ dependent manner and $\mathrm{HmsC}$ might play an important role in sensing the redox state.

To further verify whether the periplasmic redox environment affects the synthesis of c-di-GMP in vivo, we assessed intracellular c-di-GMP levels in the different $Y$. pestis mutants. Consistent with its biofilm phenotype, the $d s b A$ mutant exhibited significantly increased cellular c-di-GMP levels compared with the parent strain (Figure 2). In the absence of $d s b A$, we observed a significant increase in cellular c-di-GMP in the $h m s T$ mutant but not in the $h m s D$ mutant (Figure 2). Collectively, the above results suggest that $\mathrm{HmsC}$ might play an important role in sensing the redox state, and thereby regulate $\mathrm{HmsD}$ and control c-di-GMP levels, in turn regulating biofilm formation in Y. pestis.

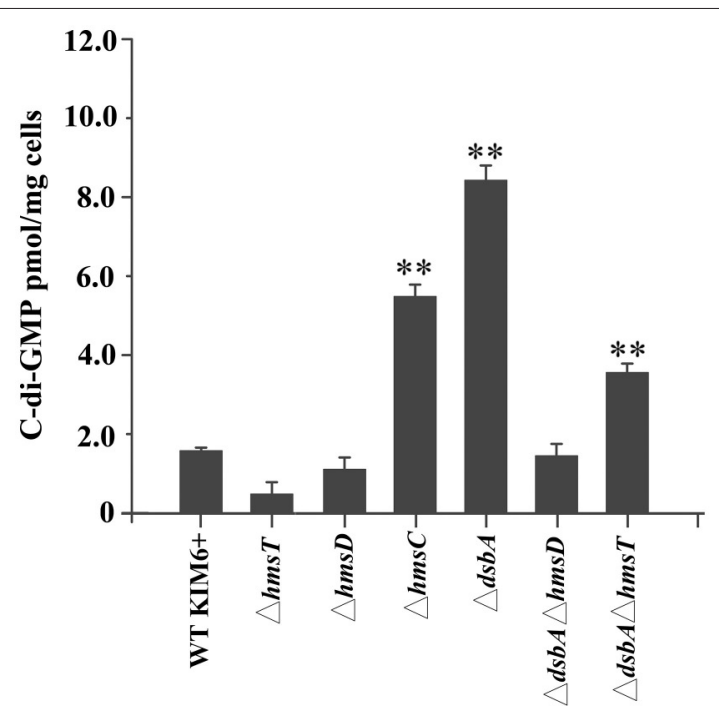

FIGURE 2 | Intracellular c-di-GMP levels in Y. pestis and its derivatives. Intracellular C-di-GMP was extracted and measured as described in the experimental procedures. ${ }^{\star \star} P<0.01$. Results are means and standard deviations of three independent experiments.
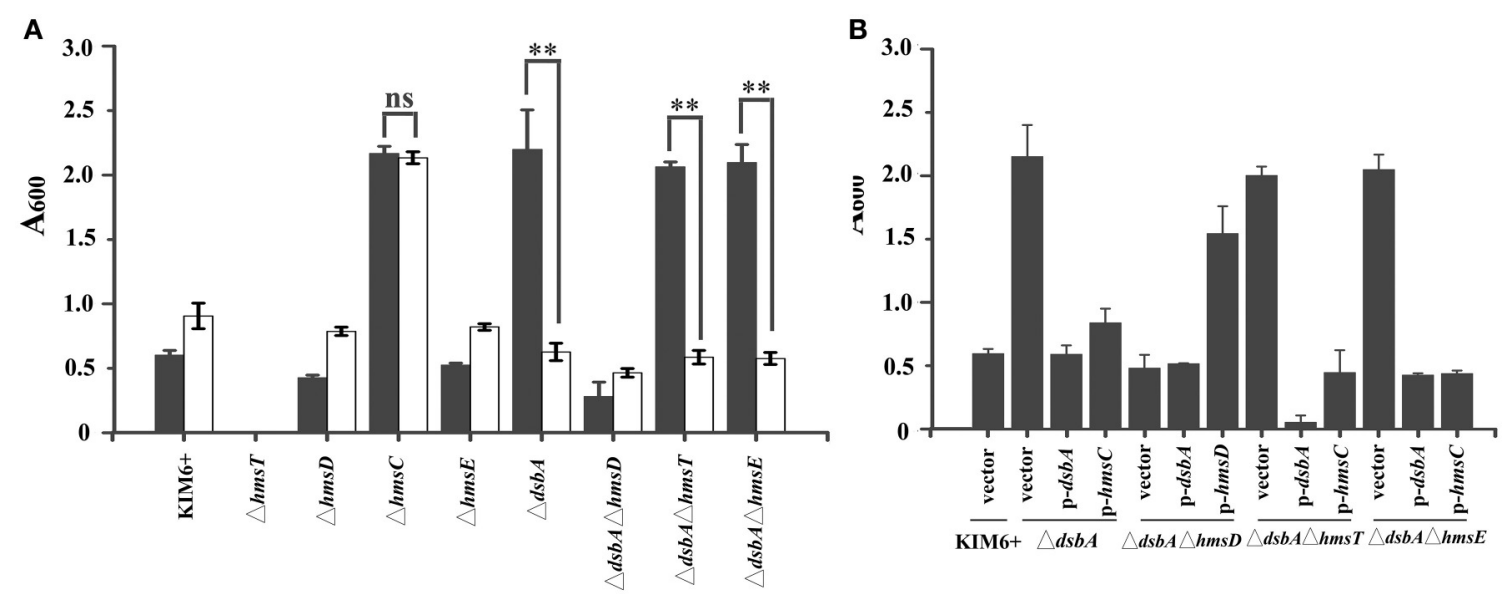

FIGURE 1 | HmsC is involved in sensing the redox environment. (A) Relative amount of adhered biofilm formed by the Yersinia pestis KIM6+ parent strain and its isogenic derivatives with (gray) or without (white) $\mathrm{CuSO}_{4}$. (B) Biofilm formation by $Y$. pestis $\mathrm{KIM} 6+, \Delta d s b A, \Delta d s b A \Delta h m s D, \Delta d s b A \Delta h m s T$, and $\Delta d s b A \Delta h m s E$ mutant strains after transformation with the empty pUC19 vector or plasmids containing $h m s C$ (pYC212), $h m s D(p Y C 211)$ or $d s b A(p Y C 257)$. ${ }^{\star \star} P<0.01$, ns $=$ not significant. Results are means and standard deviations of four independent experiments. 


\section{The Periplasmic Redox Environment Regulates HmsC Protein Levels and the Interaction between HmsC and HmsD}

The redox environment might affect the expression of $\mathrm{HmsCDE}$ or the regulatory role of $\mathrm{HmsC}$ on $\mathrm{HmsD}$ to modulate biofilm formation. To test this hypothesis, we first analyzed $\mathrm{HmsCDE}$ protein levels under different redox conditions. $\mathrm{HmsD}$ protein levels were not affected by mutation of $d s b A$ (Figure 3B), while $\mathrm{HmsC}$ was slightly decreased and $\mathrm{HmsE}$ was slightly increased in the $d s b A$ mutant (Figure 3). Meanwhile, addition of the oxidant $\mathrm{CuSO}_{4}$ stabilized $\mathrm{HmsC}$ in the $d s b A$ mutant (Figure 3A). These results suggest that the protein level of $\mathrm{HmsC}$ is affected by the periplasmic redox environment. We previously reported that $\mathrm{HmsC}$ has a direct interaction with the periplasmic region of $\mathrm{HmsD}$ and the loss of $\mathrm{HmsC}$ function releases the repression of $\mathrm{HmsD}$, in turn stimulating biofilm formation. To investigate whether the periplasmic redox environment affects the interaction between $\mathrm{HmsC}$ and $\mathrm{HmsD}$, we performed co-purification analysis of $\mathrm{HmsC}$ and the periplasmic domain of HmsD as previously described (Ren et al., 2014). As shown in Figure 4, the periplasmic domain of $\mathrm{HmsD}$ was co-purified with

A

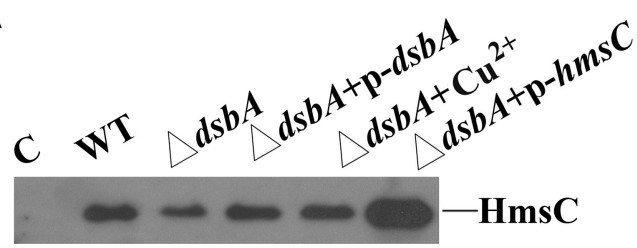

$\begin{array}{lllll}1.0 & 0.53 & 0.86 & 0.85 & 1.95\end{array}$

B
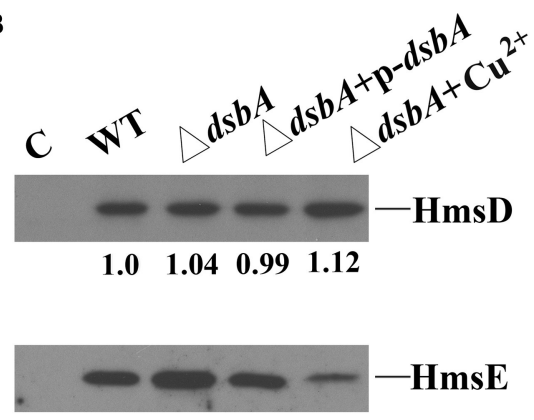

$\begin{array}{llll}1.0 & 1.61 & 1.09 & 0.31\end{array}$

FIGURE 3 | Effect of the dsbA mutant on $\mathrm{HmsC}$, HmsD, and HmsE protein levels in Y. pestis. (A) HmsC protein levels detected by western blotting. Whole cells were extracted from Y. pestis SY1540 (hmsC-Flag) and Y. pestis SY1542 ( $\Delta d s b A, h m s C$-Flag) containing plasmid pYC257 ( $\mathrm{p}-d s b A$ ) or pYC212 $(\mathrm{p}-\mathrm{hms} \mathrm{C})$, and from cells grown in LB medium supplemented with $\mathrm{CuSO}_{4}$. (B) $\mathrm{HmsD}$ and $\mathrm{HmsE}$ protein levels detected by western blotting. Whole cells were extracted from Y. pestis SY1562 (hmsD-Myc), SY2046 ( $h m s E-H A)$, Y. pestis SY1564 ( $\Delta d s b A, h m s D$-Myc), and Y. pestis SY2994 ( $\Delta d s b A$, $h m s E-H A)$ containing plasmid pYC257 ( $p-d s b A)$, and from cells supplemented with $\mathrm{CuSO}_{4} . \mathrm{HmsC}, \mathrm{HmsD}$, and $\mathrm{HmsE}$ protein levels were quantified using Image J. Numbers below blots indicate the ratio of proteins in the indicated sample compared with samples collected at mid-stationary phase (Set as 1) based on at least two independent experiments.
$\mathrm{HmsC}$ to a lesser extent in the $d s b A$ mutant than in the wild-type strain, suggesting that the interaction between $\mathrm{HmsC}$ and $\mathrm{HmsD}$ is affected by the periplasmic redox environment. Taken together, these results suggest that the redox state might regulate biofilm formation through modulation of the conformation of the HmsC protein and its interaction with $\mathrm{HmsD}$.

\section{Disulphide Bond Formation Is Critical for HmsC Function}

Sequence alignment of $\mathrm{HmsC}$ and its homolog YfiR revealed four conserved cysteines in $\mathrm{HmsC}$ (Figure S4). Analysis of the crystal structure of YfiR suggests that these cysteines form two disulphide bonds: Cys71-Cys110 (corresponding to Cys87-Cys126 in HmsC) and Cys145-Cys152 (corresponding to Cys161-Cys168 in HmsC) (Yang et al., 2015). Since disulphide bond formation is dependent on the redox environment, we hypothesized that the redox state affects disulphide bond formation, and thereby regulates the conformation of $\mathrm{HmsC}$ and its interaction with HmsD. If so, breakage of the disulphide bonds will likely affect $\mathrm{HmsC}$ function, resulting in hyper biofilm formation. To test this hypothesis, we constructed a series of $\mathrm{HmsC}$ mutants in which the four conserved cysteine were replaced with alanine. Although the Cys145-Cys152 disulphide bond in YfiR (corresponding to Cys161-Cys168 in $\mathrm{HmsC}$ ) is critical for protein folding (Yang et al., 2015), the function of $\mathrm{HmsC}$ was only partially lost when Cys161 or Cys168 was mutated (Figure 5A), whereas $\mathrm{HmsC}$ function was completely lost when Cys87 or Cys 126 was mutated (Figure 5A). This suggests that the Cys87-Cys126 disulphide bond plays a critical role in maintaining the structure and function of $\mathrm{HmsC}$.

To further determine whether breakage of disulphide bonds affects the stability of $\mathrm{HmsC}$, we analyzed the expression levels of $\mathrm{HmsC}$ mutants. $\mathrm{HmsC}$ fusion proteins containing the Flag tag, which displayed a similar phenotype to mutants of $\mathrm{HmsC}$ without the tag (Ren et al., 2014), were constructed and expressed in the Y. pestis hms C mutant. As shown in Figure 5B, breakage of the Cys161-Cys168 disulphide bond had a slight effect on $\mathrm{HmsC}$ protein levels, whereas breakage of the Cys87-Cys126

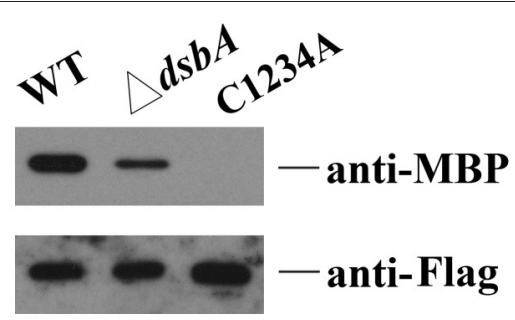

FIGURE 4 | Disulphide bond formation affects the interaction between HmsC and $\mathrm{HmsD}$. $\mathrm{HmsC}$, and $\mathrm{HmsC}$ mutants were co-purified with the periplasmic domain of $\mathrm{HmsD}$ in vivo. P-MBP-HmsD ${ }^{\mathrm{N} 49-155}$ was co-expressed with $\mathrm{HmsC}-\mathrm{His}_{8}$ in the wild-type strain (lane 1) or the dsbA mutant (lane 2). P-MBP-HmsD ${ }^{\mathrm{N} 49-155}$ was co-expressed with HmsC C87AC126AC161AC168A Flag-His 8 (C1234A) (lane 3), purified using Ni-NTA resin, and detected with anti-MBP tag and anti-Flag antibodies. 


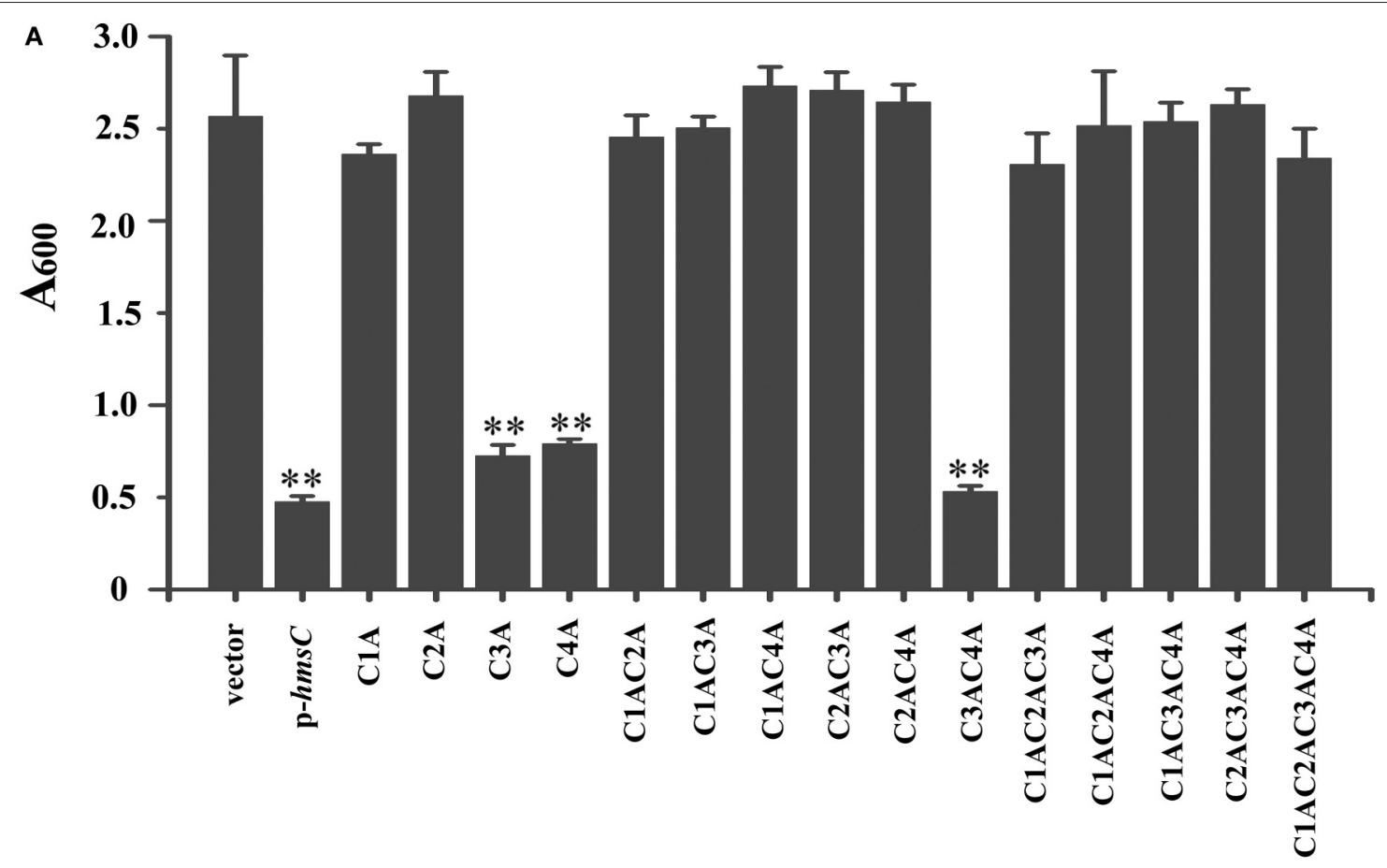

B

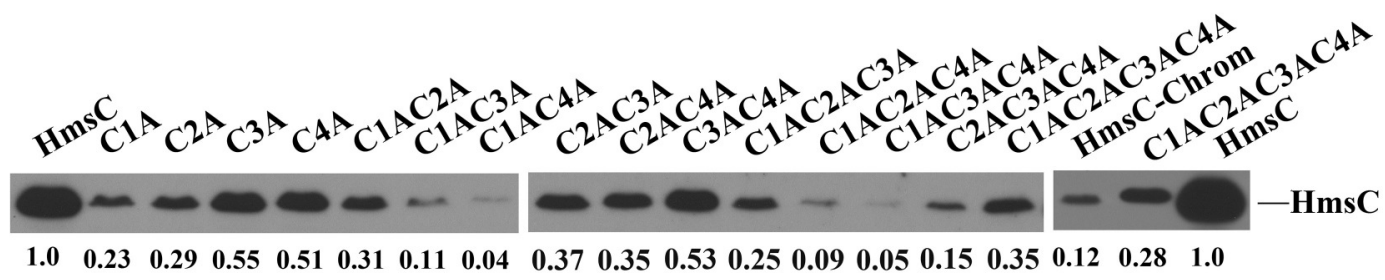

FIGURE 5 | Disulphide bonds are important for HmsC function. (A) Relative amount of adhered biofilm from the $h m s C$ mutant strain and $h m s C$ mutant strains transformed with the pUC19 vector or the plasmids containing $h m s C$ (pYC212), hms $C^{\mathrm{C} 87 \mathrm{~A}}$ (pYC316, C1A), hms $C^{\mathrm{C} 126 \mathrm{~A}}$ (pYC317, C2A), hmsC ${ }^{\mathrm{C} 161 \mathrm{~A}}$ (pYC318, C3A), hms C ${ }^{\mathrm{C} 168 \mathrm{~A}}$ (pYC317, C4A), hms C C87AC126A (pYC320, C1AC2A), hmsC C87AC161A (pYC321, C1AC3A), hmsCC87AC168A (pYC322, C1AC4A), hms C C126AC161A (pYC323, C2AC3A), hmsC C126AC168A (pYC324, C2AC4A), hmsCC161AC168A (pYC325, C3AC4A), hmsCC87AC126AC161A (pYC326,

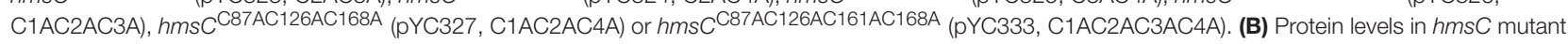
strains transformed with the empty pUC19 vector, or plasmids containing $h m s C$ (pYC212) or $h m s C$ conserved cysteine mutants. ${ }^{* *} P<0.01$. Results are means and standard deviations of four independent experiments. HmsC protein levels were quantified using Image J. Numbers below blots indicate the ratio of proteins in the indicated samples compared with samples collected at mid-stationary phase (Set as 1) based on at least two independent experiments.

disulphide bond had a much more significant effect. These results suggest that disulphide bonds are critical for maintaining the structure and function of HmsC. Surprisingly, although HmsC was non-functional when the Cys87-Cys126 disulphide bond was broken, the amount of HmsC mutant protein (Cys87 and/or Cys126) expressed from the respective plasmid was higher than the amount of wild-type $\mathrm{HmsC}$ expressed from the chromosome (Figure 5B). This suggests that disulphide bond formation affects the role of $\mathrm{HmsC}$ not only by regulating the stability of the $\mathrm{HmsC}$ protein, but also by regulating its interaction with HmsD. To verify this hypothesis, we performed co-purification analysis of $\mathrm{HmsC}$ and the periplasmic domain of HmsD. Consistent with the previous results that $d s b A$ mutation affect the co-purification of $\mathrm{HmsC}$ and $\mathrm{HmsD}$ (Figure 4), HmsC mutant (C1234A) lacking disulphide bonds could not be co-purified with $\mathrm{HmsD}$, suggesting that disulphide bond formation is crucial for the interaction between $\mathrm{HmsC}$ and $\mathrm{HmsD}$.

\section{DISCUSSION}

Bacteria are exposed to a constantly changing environment, and they are required to sense and react to these changes in order to adapt and thrive. HmsCDE and its homolog YfiBNR are involved in sensing environmental conditions and regulating biofilm formation (Malone et al., 2010, 2012; Ren et al., 2014; 

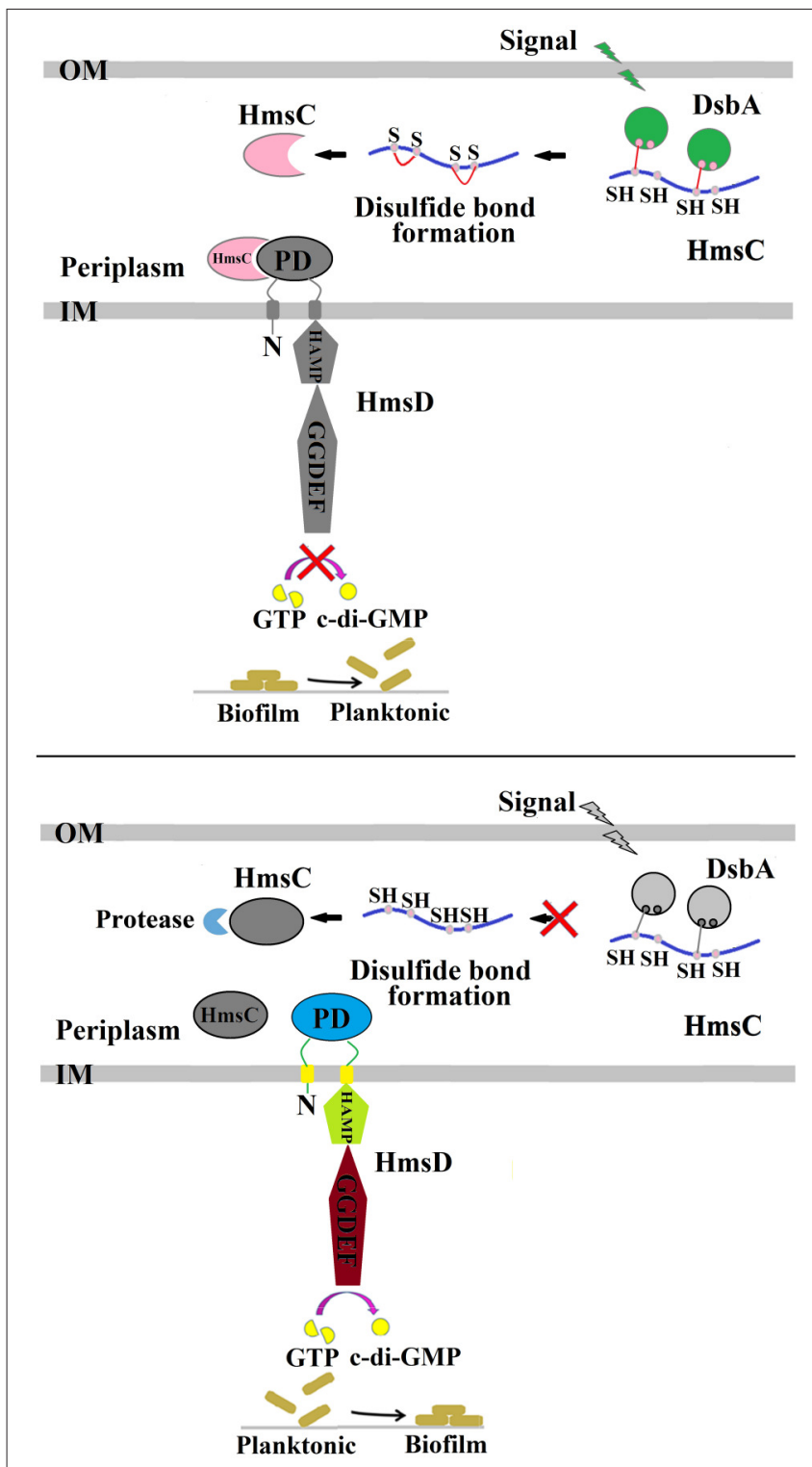

FIGURE 6 | Regulation of Y. pestis biofilm formation by DsbA and HmsC. The periplasmic protein HmsC possesses four conserved cysteines that likely form disulphide bonds that are modulated by DsbA. The formation of disulphide bonds controls HmsC protein degradation and protein conformation, and determines whether $\mathrm{HmsC}$ binds to or disassociates from the periplasmic sensor domain of $\mathrm{HmsD}$, which controls the activity of $\mathrm{HmsD}$ that in turn regulates the intracellular c-di-GMP levels and ultimately biofilm formation.

Bobrov et al., 2015). In response to cell stress, the outer membrane protein $\mathrm{YfiB} / \mathrm{HmsE}$ sequesters the periplasmic protein YfiR/HmsC, alleviating its inhibition of YfiN/HmsD in the inner membrane, and thus provoking the diguanylate cyclase activity of YfiN to induce c-di-GMP production (Malone et al., 2012; Bobrov et al., 2015). However, HmsE is not required for increased biofilm formation in a reducing environment (Ren et al., 2016). In addition, $y f i B$ is absent in some bacteria such as Salmonella and Dickeya (Malone et al., 2010, 2012), which contain fully conserved $y f i R$ and $y f i N$, suggesting that there might be another protein involved in sensing environmental signals. Herein, we report that the periplasmic protein $\mathrm{HmsC}$ plays an important role in sensing the redox state of the environment and regulation of biofilm formation.

The crystal structure of YfiR (Li et al., 2015; Yang et al., 2015), a homolog of HmsC, revealed two pairs of intraprotein disulphide bonds, Cys71-Cys110 and Cys145-Cys152, which are conserved in homologs in many bacteria (Figure S4). The disulphide bonds are important for the stability of $\mathrm{HmsC}$ and the interaction of $\mathrm{HmsC}-\mathrm{HmsD}$ (Figures 4, 5B). In addition, we found that the Cys87-Cys126 disulphide bond in $\mathrm{HmsC}$ (corresponding to Cys71-Cys110 in YfiR) might play a major role in sensing the redox state and regulate the function and stability of $\mathrm{HmsC}$. This disulphide bond can only form in oxidative conditions (Yang et al., 2015) and was found to be critical for the function of HmsC (Figure 5). By contrast, the Cys145-Cys152 disulphide bond in YfiR remained intact in the reducing environment of the cytoplasm (Yang et al., 2015) and breakage of the corresponding disulphide in $\mathrm{HmsC}$ only partially affected its function (Figure 5A).

The formation of disulphide bonds in $\mathrm{HmsC}$ might be modulated in response to changes in the redox environment in the periplasm, which in turn could regulate the stability of the $\mathrm{HmsC}$ protein and its interaction with $\mathrm{HmsD}$. Further modulation via $\mathrm{HmsC}$ inhibition might regulate the diguanylate cyclase activity of $\mathrm{HmsD}$ and thus control biofilm formation (Figure 6). The redox state in the periplasm can be affected by substances such as DTT and $\mathrm{Cu}^{2+}$ that generate reducing and oxidizing conditions, respectively. However, the periplasmic redox environment is mainly controlled by the DsbA/B system, which coordinates disulphide bond formation in periplasmic proteins. Thus, environmental signals could modulate the DsbA/B system, which in turn regulates HmsC and controls biofilm formation.

\section{AUTHOR CONTRIBUTIONS}

Experiment designation: GR and YS; Experiment carry out: GR and XG; Manuscript writing: GR and YS; Manuscript review and modification: GR and YS.

\section{FUNDING}

This work was supported by the National Major Research \& Development Program of China (2016YFC1202600), the National Basic Research Program of China (973 Program) (2015CB554200), the National Natural Science Foundation of China (81501723) and (31670139) and CAMS Initiative for Innovative Medicine (2016-I2M-1-013).

\section{SUPPLEMENTARY MATERIAL}

The Supplementary Material for this article can be found online at: http://journal.frontiersin.org/article/10.3389/fcimb.2017. 00355/full\#supplementary-material 


\section{REFERENCES}

Anwar, N., Rouf, S. F., Romling, U., and Rhen, M. (2014). Modulation of biofilm-formation in Salmonella enterica serovar Typhimurium by the periplasmic DsbA/DsbB oxidoreductase system requires the GGDEF-EAL domain protein STM3615. PLoS ONE 9:e106095. doi: 10.1371/journal.pone.01 06095

Bardwell, J. C. (1994). Building bridges: disulphide bond formation in the cell. Mol. Microbiol. 14, 199-205. doi: 10.1111/j.1365-2958.1994.tb 01281.x

Bardwell, J. C., Lee, J. O., Jander, G., Martin, N., Belin, D., and Beckwith, J. (1993). A pathway for disulfide bond formation in vivo. Proc. Natl. Acad. Sci. U.S.A. 90, 1038-1042. doi: $10.1073 /$ pnas. 90.3 .1038

Battistoni, A., Mazzetti, A. P., and Rotilio, G. (1999). In vivo formation of $\mathrm{Cu}$, Zn superoxide dismutase disulfide bond in Escherichia coli. FEBS Lett. 443, 313-316. doi: 10.1016/S0014-5793(98)01725-6

Bellows, L. E., Koestler, B. J., Karaba, S. M., Waters, C. M., and Lathem, W. W. (2012). Hfq-dependent, co-ordinate control of cyclic diguanylate synthesis and catabolism in the plague pathogen Yersinia pestis. Mol. Microbiol. 86, 661-674. doi: $10.1111 / \mathrm{mmi} .12011$

Bobrov, A. G., Kirillina, O., Ryjenkov, D. A., Waters, C. M., Price, P. A., Fetherston, J. D., et al. (2011). Systematic analysis of cyclic di-GMP signalling enzymes and their role in biofilm formation and virulence in Yersinia pestis. Mol. Microbiol. 79, 533-551. doi: 10.1111/j.1365-2958.2010. 07470.x

Bobrov, A. G., Kirillina, O., Vadyvaloo, V., Koestler, B. J., Hinz, A. K., Mack, D., et al. (2015). The Yersinia pestis HmsCDE regulatory system is essential for blockage of the oriental rat flea (Xenopsylla cheopis), a classic plague vector. Environ. Microbiol. 17, 947-959. doi: 10.1111/1462-2920. 12419

Datsenko, K. A., and Wanner, B. L. (2000). One-step inactivation of chromosomal genes in Escherichia coli K-12 using PCR products. Proc. Natl. Acad. Sci. U.S.A. 97, 6640-6645. doi: 10.1073/pnas.120163297

Eisen, R. J., Bearden, S. W., Wilder, A. P., Montenieri, J. A., Antolin, M. F., and Gage, K. L. (2006). Early-phase transmission of Yersinia pestis by unblocked fleas as a mechanism explaining rapidly spreading plague epizootics. Proc. Natl. Acad. Sci. U.S.A. 103, 15380-15385. doi: 10.1073/pnas.0606 831103

Eisen, R. J., and Gage, K. L. (2009). Adaptive strategies of Yersinia pestis to persist during inter-epizootic and epizootic periods. Vet. Res. 40:1. doi: 10.1051/vetres:2008039

Eisen, R. J., Wilder, A. P., Bearden, S. W., Montenieri, J. A., and Gage, K. L. (2007). Early-phase transmission of Yersinia pestis by unblocked Xenopsylla cheopis (Siphonaptera: Pulicidae) is as efficient as transmission by blocked fleas. J. Med. Entomol. 44, 678-682. doi: 10.1093/jmedent/44.4.678

Hufnagel, D. A., DePas, W. H., and Chapman, M. R. (2014). The disulfide bonding system suppresses CsgD-independent cellulose production in Escherichia coli. J. Bacteriol. 196, 3690-3699. doi: 10.1128/JB.02019-14

Jones, H. A., Lillard, J. W. Jr., and Perry, R. D. (1999). HmsT, a protein essential for expression of the haemin storage (Hms+) phenotype of Yersinia pestis. Microbiology 145 (Pt 8), 2117-2128. doi: 10.1099/13500872-145-8-2117

Kirillina, O., Fetherston, J. D., Bobrov, A. G., Abney, J., and Perry, R. D. (2004). $\mathrm{HmsP}$, a putative phosphodiesterase, and HmsT, a putative diguanylate cyclase, control Hms-dependent biofilm formation in Yersinia pestis. Mol. Microbiol. 54, 75-88. doi: 10.1111/j.1365-2958.2004.04253.x
Li, S., Li, T., Xu, Y., Zhang, Q., Zhang, W., Che, S., et al. (2015). Structural insights into YfiR sequestering by YfiB in Pseudomonas aeruginosa PAO1. Sci. Rep. 5:16915. doi: 10.1038/srep16915

Malone, J. G., Jaeger, T., Manfredi, P., Dotsch, A., Blanka, A., Bos, R., et al. (2012). The YfiBNR signal transduction mechanism reveals novel targets for the evolution of persistent Pseudomonas aeruginosa in cystic fibrosis airways. PLoS Pathog. 8:e1002760. doi: 10.1371/journal.ppat.1002760

Malone, J. G., Jaeger, T., Spangler, C., Ritz, D., Spang, A., Arrieumerlou, C., et al. (2010). YfiBNR mediates cyclic di-GMP dependent small colony variant formation and persistence in Pseudomonas aeruginosa. PLoS Pathog. 6:e1000804. doi: 10.1371/journal.ppat.1000804

Nakamoto, H., and Bardwell, J. C. (2004). Catalysis of disulfide bond formation and isomerization in the Escherichia coli periplasm. Biochim. Biophys. Acta 1694, 111-119. doi: 10.1016/j.bbamcr.2004.02.012

Ren, G. X., Fan, S., Guo, X. P., Chen, S., and Sun, Y. C. (2016). Differential regulation of c-di-GMP metabolic enzymes by environmental signals modulates biofilm formation in Yersinia pestis. Front. Microbiol. 7:821. doi: $10.3389 /$ fmicb.2016.00821

Ren, G. X., Yan, H. Q., Zhu, H., Guo, X. P., and Sun, Y. C. (2014). HmsC, a periplasmic protein, controls biofilm formation via repression of $\mathrm{HmsD}$, a diguanylate cyclase in Yersinia pestis. Environ. Microbiol. 16, 1202-1216. doi: 10.1111/1462-2920.12323

Ryan, R. P., Fouhy, Y., Lucey, J. F., Crossman, L. C., Spiro, S., He, Y. W., et al. (2006). Cell-cell signaling in Xanthomonas campestris involves an HD-GYP domain protein that functions in cyclic di-GMP turnover. Proc. Natl. Acad. Sci. U.S.A. 103, 6712-6717. doi: 10.1073/pnas.0600345103

Ryjenkov, D. A., Tarutina, M., Moskvin, O. V., and Gomelsky, M. (2005). Cyclic diguanylate is a ubiquitous signaling molecule in bacteria: insights into biochemistry of the GGDEF protein domain. J. Bacteriol. 187, 1792-1798. doi: 10.1128/JB.187.5.1792-1798.2005

Schmidt, A. J., Ryjenkov, D. A., and Gomelsky, M. (2005). The ubiquitous protein domain EAL is a cyclic diguanylate-specific phosphodiesterase: enzymatically active and inactive EAL domains. J. Bacteriol. 187, 4774-4781. doi: 10.1128/JB.187.14.4774-4781.2005

Sun, Y. C., Hinnebusch, B. J., and Darby, C. (2008). Experimental evidence for negative selection in the evolution of a Yersinia pestis pseudogene. Proc. Natl. Acad. Sci. U.S.A. 105, 8097-8101. doi: 10.1073/pnas.0803525105

Sun, Y. C., Koumoutsi, A., Jarrett, C., Lawrence, K., Gherardini, F. C., Darby, C., et al. (2011). Differential control of Yersinia pestis biofilm formation in vitro and in the flea vector by two c-di-GMP diguanylate cyclases. PLoS ONE 6:e19267. doi: 10.1371/journal.pone.0019267

Yang, X., Yang, X. A., Xu, M., Zhou, L., Fan, Z., and Jiang, T. (2015). Crystal structures of YfiR from Pseudomonas aeruginosa in two redox states. Biochem. Biophys. Res. Commun. 461, 14-20. doi: 10.1016/j.bbrc.2015.03.160

Conflict of Interest Statement: The authors declare that the research was conducted in the absence of any commercial or financial relationships that could be construed as a potential conflict of interest.

Copyright $\odot 2017$ Ren, Guo and Sun. This is an open-access article distributed under the terms of the Creative Commons Attribution License (CC BY). The use, distribution or reproduction in other forums is permitted, provided the original author(s) or licensor are credited and that the original publication in this journal is cited, in accordance with accepted academic practice. No use, distribution or reproduction is permitted which does not comply with these terms. 